\title{
BOGOMOLOV-GIESEKER INEQUALITY AND COHOMOLOGY VANISHING IN CHARACTERISTIC $p$
}

\author{
TOHRU NAKASHIMA
}

(Communicated by Eric Friedlander)

\begin{abstract}
We prove an analogue of the Bogomolov-Gieseker inequality for rank-two bundles on varieties defined over a field of positive characteristic. We derive from this some vanishing results for cohomology of line bundles.
\end{abstract}

\section{INTRODUCTION}

Let $X$ be a smooth projective variety defined over an algebraically closed field $k$ of characteristic $p>0$. In [M2], a Bogomolov-Gieseker type inequality for rank-two bundles on $X$ has been obtained under the assumption that $X$ is not uniruled. In the present note we shall show that a similar inequality holds under certain stability conditions on the tangent bundle of $X$. As a corollary, we obtain some vanishing results for cohomology of line bundles. We also consider the vanishing for bundles of higher rank.

\section{MAIN ReSUlT}

In what follows, we assume that all varieties are defined over an algebraically closed field $k$ of characteristic $p>0$.

Let $X$ be a smooth projective variety over $k$, and let $H$ be an ample line bundle on $X$. Let $E$ be a vector bundle on $X$. Following [M1], we say that $E$ is $p$-semistable with respect to $H$ if, for all $m \geq 0$, the $m$-th iterated Frobenius pull-back $\left(F^{m}\right)^{*} E$ is $\mu$-semistable with respect to $H$. We have the following Bogomolov-Gieseker type inequality, which is due to A. Moriwaki.

Proposition 1 ([M1,Theorem 1]). Let $X$ be a smooth projective variety of dimension $d \geq 2$, and let $H$ be an ample line bundle on $X$. Let $E$ be a rank-two vector bundle on $X$ which is p-semistable with respect to $H$. Then we have

$$
\left\{c_{1}(E)^{2}-4 c_{2}(E)\right\} . H^{d-2} \leq 0 .
$$

Let $X, H$ be as above. For a vector bundle $E$ on $X$, we denote by $\mu_{H}(E)$ the slope of $E$ with respect to $H$ :

$$
\mu_{H}(E)=\frac{c_{1}(E) \cdot H^{d-1}}{\mathrm{rk} E} .
$$

Received by the editors June 7, 1994.

1991 Mathematics Subject Classification. Primary 14F17; Secondary 14J60. 
Let $T_{X}$ be the tangent bundle of $X$, and let

$$
0=T_{0} \subset T_{1} \subset \cdots \subset T_{l-1} \subset T_{l}=T_{X}
$$

be the Harder-Narasimhan filtration of $T_{X}$ with respect to $H$. We set $\mu_{H}(X):=$ $\mu_{H}\left(T_{X} / T_{l-1}\right)$. The following is a generalization of [L-S, 2.4.Satz].

Lemma 1. Let $X$ be a smooth projective variety of dimension $d \geq 1$ with an ample line bundle $H$. Let $E$ be a rank-two vector bundle on $X$. Assume that $E$ is $\mu$-semistable and $F^{*} E$ is not $\mu$-semistable with respect to $H$. If $M \subset F^{*} E$ denotes the maximal destabilizing subline bundle, then we have

$$
M . H^{d-1} \leq \frac{1}{2}\left\{p c_{1}(E) \cdot H^{d-1}-\mu_{H}(X)\right\} .
$$

Proof. By the radical descent theory in [G], we have an $\mathscr{O}_{X}$-homomorphism

$$
f: T_{X} \rightarrow \operatorname{End}_{\mathscr{O}_{X^{(p)}}}\left(F^{*} E\right)
$$

where $X^{(p)}$ is the scheme obtained from the base change by the Frobenius map of $k$. Composing $f$ with the inclusion $M \hookrightarrow F^{*} E$ and with the projection $F^{*} E \rightarrow F^{*} E / M$, we obtain the following $\mathscr{O}_{X}$-homomorphism

$$
\tilde{f}: T_{X} \rightarrow \operatorname{Hom}_{\mathscr{O}_{X}}\left(M, F^{*} E / M\right) .
$$

We claim that $\tilde{f} \neq 0$. Otherwise, there would exist a subsheaf $M^{\prime} \subset E$ such that $F^{*} M^{\prime}=M$, contradicting the semistability of $E$. Hence the desired inequality follows immediately.

Theorem 1. Let $X$ be a smooth projective variety of dimension $d \geq 2$ with an ample line bundle $H$. Let $E$ be a rank-two vector bundle on $X$, which is $\mu$-semistable with respect to $H$.

(1) If $\mu_{H}(X) \geq 0$, then $\left\{c_{1}(E)^{2}-4 c_{2}(E)\right\} \cdot H^{d-2} \leq 0$.

(2) If $\mu_{H}(X)<0$, then

$$
\left\{c_{1}(E)^{2}-4 c_{2}(E)\right\} \cdot H^{d-2} \leq \frac{\mu_{H}(X)^{2}}{p^{2} H^{d}} .
$$

Proof. Let $m$ be the smallest integer such that $\left(F^{m}\right)^{*} E$ is not $\mu$-semistable. We claim that if $\mu_{H}(X) \geq 0$, then we have $m=\infty$. Indeed, assume that $m<\infty$ and let $M^{\prime}$ be the maximal destabilizing subsheaf of $\left(F^{m}\right)^{*} E$. If we define the $\mathbb{Q}$-line bundle $M:=M^{\prime} / p^{m}$, then

$$
\frac{c_{1}(E) \cdot H^{d-1}}{2}<M \cdot H^{d-1} .
$$

Applying Lemma 1 to $\left(F^{m-1}\right)^{*} E$, we obtain

$$
M . H^{d-1} \leq \frac{1}{2}\left\{c_{1}(E) \cdot H^{d-1}-\frac{\mu_{H}(X)}{p^{m}}\right\},
$$

which is a contradiction if $\mu_{H}(X) \geq 0$. Hence in case (1) we are done by Proposition 1.

Assume that $\mu_{H}(X)<0$. If we set

$$
\alpha=\frac{\left(2 M-c_{1}(E)\right) \cdot H^{d-1}}{H^{d}},
$$


then we have

$$
0<\alpha \leq-\frac{\mu_{H}(X)}{p^{m} H^{d}}
$$

Since $\left(2 M-c_{1}(E)-\alpha H\right) \cdot H^{d-1}=0$, the Hodge index theorem yields

$$
\left(2 M-c_{1}(E)-\alpha H\right)^{2} \cdot H^{d-2} \leq 0 .
$$

Therefore we have

$$
\begin{aligned}
\left\{c_{1}(E)^{2}-4 c_{2}(E)\right\} \cdot H^{d-2} & \leq\left(2 M-c_{1}(E)\right)^{2} \cdot H^{d-2} \\
& \leq\left\{2 \alpha\left(2 M-c_{1}(E)\right) \cdot H-\alpha^{2} H^{2}\right\} \cdot H^{d-2} \\
& =\alpha^{2} H^{d} \\
& \leq \frac{\mu_{H}(X)^{2}}{p^{2 m} H^{d}} \leq \frac{\mu_{H}(X)^{2}}{p^{2} H^{d}} .
\end{aligned}
$$

This completes the proof.

The above theorem implies vanishing results for the cohomology of line bundles as in [M2].

Corollary 1. Let $X$ be a smooth projective variety of dimension $d \geq 2$ with an ample line bundle $H$. Let $L$ be a nef line bundle on $X$. Assume that either

(1) $\mu_{H}(X) \geq 0$ and $L^{2} \cdot H^{d-2}>0$, or

(2) $\mu_{H}(X)<0$ and

$$
L^{2} \cdot H^{d-2}>\frac{\mu_{H}(X)^{2}}{p^{2} H^{d}} .
$$

Then we have $H^{1}\left(X, L^{-1}\right)=0$.

Proof. If $H^{1}\left(X, L^{-1}\right) \neq 0$, then we obtain a non-split extension

$$
0 \rightarrow \mathscr{O}_{X} \rightarrow E \rightarrow L \rightarrow 0 .
$$

If we show that $E$ is $\mu$-semistable with respect to $H$, then we obtain a contradiction by Theorem 1 , since we have $\left\{c_{1}(E)^{2}-4 c_{2}(E)\right\} \cdot H^{d-2}=L^{2} \cdot H^{d-2}$. To show the $\mu$-semistability of $E$, assume that there exist a subline bundle $M \hookrightarrow E$ with

$(2 M-L) \cdot H^{d-1}>0$ and an exact sequence

$$
0 \rightarrow M \rightarrow E \rightarrow \mathscr{I}_{Z}(L-M) \rightarrow 0
$$

where $Z$ is a codimension-two subscheme. The composition map $M \hookrightarrow E \rightarrow L$ is not zero, since otherwise we would obtain a nontrivial map $M \rightarrow \mathscr{\sigma}_{X}$, which is impossible. Hence there exists an effective divisor $D$ which is linearly equivalent to $L-M$ and satisfies $(L-2 D) \cdot H^{d-1}>0$. Then, by the Hodge index theorem, we have $(L-2 D) \cdot D \cdot H^{d-2}>0$. On the other hand, we have $Z \cdot H^{d-2}=c_{2}(E(-M)) \cdot H^{d-2}=(D-L) \cdot D \cdot H^{d-2} \geq 0$. It follows that $D=0$ or, equivalently, $L=M$, hence the original sequence must split. This contradiction proves that $E$ is $\mu$-semistable.

We say that $T_{X}$ is nef if the tautological line bundle $\mathscr{O}(1)$ on $\mathbb{P}\left(T_{X}\right)$ is nef. For example, $T_{X}$ is nef if it is globally generated. 
Corollary 2. Let $X$ be a smooth projective variety of dimension $d \geq 2$ with nef tangent bundle $T_{X}$. If $L$ is a nef and big line bundle on $X$, then we have $H^{1}\left(X, L^{-1}\right)=0$.

Proof. Let $Q$ be a quotient bundle of $T_{X}$. It can be easily seen that if $T_{X}$ is nef, then $c_{1}(Q) \cdot H^{d-1} \geq 0$ for every ample line bundle $H$. In particular, we have $\mu_{H}(X) \geq 0$. Hence the claim follows from Corollary 1 .

Corollary 3. Let $X$ be a Fano variety of dimension $d \geq 2$ such that $T_{X}$ is $\mu$-semistable with respect to $-K_{X}$. Then we have $H^{1}\left(X, m K_{X}\right)=0$ for all $m>0$.

\section{VANISHING FOR BUNDLES OF HIGHER RANK}

Let $X$ be a smooth projective variety. A vector bundle $E$ on $X$ is said to be cohomologically p-ample if, for every coherent sheaf $\mathscr{F}$ on $X$, there exists an integer $m_{0}=m_{0}(\mathscr{F})$ such that for all $m \geq m_{0}$ and all $i>0$ we have $H^{i}\left(X, \mathscr{F} \otimes\left(F^{m}\right)^{*} E\right)=0$. It is known that every cohomologically $p$-ample vector bundle is ample. A line bundle is cohomologically $p$-ample if and only if it is ample (cf. $[\mathrm{K}])$.

Proposition 2. Let $X$ be a smooth projective variety of dimension $d \geq 1$ with an ample line bundle $H$. Let $E$ be a vector bundle on $X$ which is cohomologically p-ample and p-semistable with respect to $H$. Assume that either

(1) $\mu_{H}(X) \geq 0$, or

(2) $\mu_{H}(X)<0$ and $\mu_{H}(E)>-\mu_{H}(X)$.

Then we have $H^{1}\left(X, E^{\vee}\right)=0$.

Proof. We define

$$
B_{X}:=\operatorname{Im}\left(d: F_{*} \mathscr{O}_{X} \rightarrow F_{*} \Omega_{X}^{1}\right)
$$

where $d$ is the differential map. Let $\mathscr{O}_{X} \rightarrow F_{*} \mathscr{O}_{X}$ be the natural map which sends $f$ to $f^{p}$. Then we have the exact sequence

$$
0 \rightarrow \mathscr{O}_{X} \rightarrow F_{*} \mathscr{O}_{X} \rightarrow B_{X} \rightarrow 0 .
$$

By assumption, it is easy to see that $\mu_{H}(X)>\mu_{H}\left(\left(F^{m}\right)^{*} E^{\vee}\right)$ for all $m \geq 0$. It follows that $H^{0}\left(X,\left(F^{m}\right)^{*} E^{\vee} \otimes \Omega_{X}^{1}\right)=\operatorname{Hom}\left(T_{X},\left(F^{m}\right)^{*} E^{\vee}\right)=0$, hence we have $H^{0}\left(X,\left(F^{m}\right)^{*} E^{\vee} \otimes B_{X}\right)=0$. Tensoring $(*)$ with $\left(F^{m}\right)^{*} E^{\vee}$ and taking cohomology, we obtain injections $H^{1}\left(X, E^{\vee}\right) \hookrightarrow H^{1}\left(X,\left(F^{m}\right)^{*} E^{\vee}\right)$ for all $m \geq 0$. On the other hand, since $E$ is cohomologically $p$-ample, we have $H^{1}\left(X,\left(F^{m}\right)^{*} E^{\vee}\right)=H^{d-1}\left(X,\left(F^{m}\right)^{*} E \otimes \omega_{X}\right)=0$ for sufficiently large $m$. Therefore, by descending induction on $m$, we obtain $H^{1}\left(X, E^{\vee}\right)=0$.

If the exact sequence $(*)$ splits, then $X$ is called Frobenius split. It has been proved that Schubert varieties are Frobenius split ([M-R]). We have a stronger vanishing result for varieties which are Frobenius split.

Proposition 3. Assume that $X$ is Frobenius split and $E$ is a cohomologically $p$ ample vector bundle on $X$. Then we have $H^{i}\left(X, E^{\vee}\right)=0$ for $i<d=\operatorname{dim} X$.

Proof. Since the exact sequence

$$
0 \rightarrow\left(F^{m}\right)^{*} E^{\vee} \rightarrow\left(F^{m}\right)^{*} E^{\vee} \otimes F_{*} \mathscr{O}_{X} \rightarrow\left(F^{m}\right)^{*} E^{\vee} \otimes B_{X} \rightarrow 0
$$


splits, we obtain injections $H^{i}\left(X,\left(F^{m}\right)^{*} E^{\vee}\right) \hookrightarrow H^{i}\left(X,\left(F^{m+1}\right)^{*} E^{\vee}\right)$ for all $m$ and $i$. Then a similar argument as in Proposition 2 completes the proof.

\section{ACKNOWLEDGMENT}

The author is very grateful to the referee for pointing out several mistakes in the first version of this paper.

\section{REFERENCES}

[G] A. Grothendieck, Technique de descente et théorèmes d'existence en géometrie algébrique I, Séminaire Bourbaki 1959/60.

[K] S. Kleiman, Ample vector bundles on algebraic surfaces, Proc. Amer. Math. Soc. 21 (1969), 673-676.

[L-S] H. Lange and U. Stuhler, Vektorbündel auf Kurven und Darstellungen der algebraischen Fundamentalgruppe, Math. Z. 156 (1977), 73-83.

[M1] A. Moriwaki, A note on Bogomolov-Gieseker's inequality in positive characteristic, Duke Math. J. 64 (1991), 361-375.

[M2] _ Frobenius pull-back of vector bundles of rank 2 over non-uniruled varieties, Math. Ann. 296 (1993), 441-451.

[M-R] V. B. Mehta and A. Ramanathan, Frobenius splitting and cohomology vanishing for Schubert varieties, Ann. of Math. (2) 122 (1985), 27-40.

Department of Mathematics, Tokyo Metropolitan University, Minami-Ohsawa 1-1, HACHIOJI-SHI, TOKYO, 192-03, JAPAN

E-mail address: nakasima@math.metro-u.ac.jp 